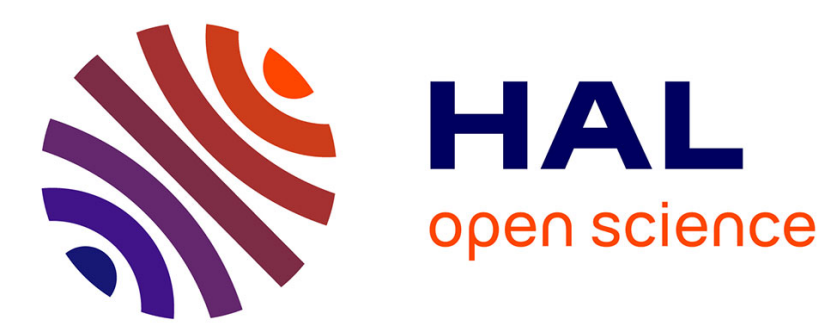

\title{
Interacting rigid polyelectrolytes
}

Jean-Louis Barrat, Jean-François Joanny

\section{To cite this version:}

Jean-Louis Barrat, Jean-François Joanny. Interacting rigid polyelectrolytes. Journal de Physique II, 1994, 4 (7), pp.1089-1102. 10.1051/jp2:1994188 . jpa-00248030

\section{HAL Id: jpa-00248030 https://hal.science/jpa-00248030}

Submitted on 1 Jan 1994

HAL is a multi-disciplinary open access archive for the deposit and dissemination of scientific research documents, whether they are published or not. The documents may come from teaching and research institutions in France or abroad, or from public or private research centers.
L'archive ouverte pluridisciplinaire HAL, est destinée au dépôt et à la diffusion de documents scientifiques de niveau recherche, publiés ou non, émanant des établissements d'enseignement et de recherche français ou étrangers, des laboratoires publics ou privés. 
Classification

Physics Abstracts

$36.20 \mathrm{C}-61.25 \mathrm{H}$

\title{
Interacting rigid polyelectrolytes
}

\author{
Jean-Louis Barrat $\left({ }^{1}\right)$ and Jean-Francois Joanny $\left({ }^{2}\right)$
}

$\left({ }^{1}\right)$ Laboratoire de Physique $\left({ }^{\star}\right)$, Ecole Normale Supérieure de Lyon, 46 Allée d'Italie, 69007 Lyon, France

$\left({ }^{2}\right)$ Institut Charles Sadron $\left({ }^{\star}\right), 6$ rue Boussingault, 67083 Strasbourg Cedex, France

(Received 15 February 1994, accepted in final form 11 April 1994)

Résumé. - Nous proposons une nouvelle description des solutions semi-diluées de polyélectrolytes rigides. Cette description se fonde sur quelques résultats analytiques obtenus pour un modèle simplifié à deux dimensions. Les solutions sans sel et avec sel ajouté ont des comportements différents. En présence de sel, la longueur de persistance d'une chaîne est peu affectée par ses interactions avec les autres chaînes, comme l'avait postulé Odijk. Dans les solutions sans sel, au contraire, la longueur de persistence varie comme la distance entre chaînes, qui dans ce cas est identique à la longueur d'écran. Ce comportement est similaire à celui qui avait été prévu par Witten et Pincus. Cependant, contrairement à ces auteurs, nous prédisons une transition très rapide entre les deux régimes.

\begin{abstract}
A new description of semidilute solutions of rigid polyelectralyte chains is proposed. The description is based on analytical results obtained for a simple two-dimensional model. These results are then transposed to the physically relevant case of interacting chains in three dimensions. Different behaviours are obtained for salt-free solutions and solutions with added salt. In the latter, the persistence length of a chain is predicted to be only weakly affected by interchain interactions, as postulated by Odijk. In salt free solutions, on the contrary, the persistence length should scale as the interchain distance, which in this case is also the screening length. This behaviour resembles the one predicted earlier by Witten and Pincus. The crossover between these two different regimes, however, is expected to be very sharp, in contrast with their prediction.
\end{abstract}

\section{Introduction.}

In this note, we summarize some ideas and results concerning the structure of interacting polyelectrolyte chains. We consider only "rigid" chains, in the sense of reference [1] which means that one isolated chain is well-described by the theory of Odijk [2] or Skolnick and

(*) (URA CNRS 1325)

$\left.{ }^{(\star \star}\right)$ (UPR CNRS 022) 
Fixman [3]. This theory is summarized as follows: if a wormlike chain with a bare persistence length $\ell_{0}$ carries a linear density $1 / A$ of monomers that interact through the long range potential $v(r)$, then the chain conformation at large length scales can be characterized by a persistence length

$$
\ell_{\mathrm{p}}=\ell_{0}+\frac{\hat{v}(k=0)}{16 \pi k_{\mathrm{B}} T A^{2}}
$$

where $\hat{v}(k)$ is the Fourier transform of the interaction potential. In the case where $v$ is the electrostatic Debye-Hückel potential, $v_{\mathrm{DH}}(r)=k_{\mathrm{B}} T\left(\ell_{\mathrm{B}} / r\right) \exp (-\kappa r), \hat{v}_{\mathrm{DH}}(k=0)=4 \pi \ell_{\mathrm{B}} / \kappa^{2}$, ( $\ell_{B}$ is the so-called Bjerrum length), equation (1) gives the electrostatic contribution to the persistence length obtained by Odijk and Skolnick and Fixman $\ell_{\mathrm{OSF}}=\ell_{\mathrm{B}} / 4 \kappa^{2} A^{2}$.

A straightforward extension of this approach to semidilute solutions could be made, in principle, by focusing on a given chain and replacing the effect of the other chains by an effective pair potential between its charged monomers. The effective (or screened) potential, unfortunately, can easily be obtained only at the level of linear response theory. At this level, it is related to the (charged monomer-charged monomer) structure factor of the semidilute solution $S(k)$ by $\hat{v}_{\text {eff }}(k)=\hat{v}(k)(1-c S(k) \hat{v}(k))$, where $c$ is the concentration of charged monomers. Equation (1) yields the persistence length

$$
\ell_{\mathrm{p}}=\ell_{0}+\frac{\hat{v}(k=0)}{16 \pi k_{\mathrm{B}} T A^{2}}(1-c S(k=0) \hat{v}(k=0))
$$

In the case of Debye-Hückel interactions the persistence length is $\ell_{\mathrm{p}}=\ell_{0}+\ell_{\mathrm{OSF}}\left(1-4 \pi \ell_{\mathrm{B}} c S(k=\right.$ $\left.0) / \kappa^{2}\right)$. The determination of the persistence length would thus only require the knowledge of the structure factor, which is a thermodynamic property of the semidilute solution and is simply related to the osmotic compressibility. It should also be noted that the interchain interactions lead to a reduction of the persistence length, that tends to balance the electrostatic rigidity created by the intra-chain interactions. In the following, we shall however see that, although this result is qualitatively correct, (i.e. the persistence length in solution is always smaller than $\left.\ell_{0}+\ell_{\mathrm{OSF}}\right)$, the use of linear response theory is not justified in general, so that in practice the usefulness of this approach is limited.

Two other theories have at present been proposed to describe semidilute solutions of stiff chains. Odijk [4] has argued that the structure of a given chain in the solution can be described by equation (1), or, in other words, that the single chain structure is not affected by the interchain interactions. A completely different picture has been proposed by Witten and Pincus [5]. In their description, the interchain interactions result in a significant reduction of the persistence length. According to them, in a salt-free solution, the persistence length is equal to the interchain distance (which in that case is also of the order of the Debye screening length). As salt is added to the solution, the persistence length increases as

$$
\ell_{\mathrm{WP}}=\frac{\kappa}{c A}
$$

until it reaches Odijk's value.

We present in the following a discussion of interacting polyelectrolytes that consists of two parts. We first study a simple two-dimensional system made up of one charged polyelectrolyte chain interacting with a liquid, or a gas, of point obstacles. In order to make contact with the actual, three-dimensional, problem, these obstacles can be thought of as a crude representation of the chains that are cut by the plane containing the tagged chain. Some exact results for this model are obtained, and compared to approximate calculations and heuristic arguments, whose validity can therefore be assessed. We then use for discussing three-dimensional systems 
an approximate calculation which has proved to work well on the simplified two-dimensional model.

The picture that emerges from our calculation is intermediate between the extremes proposed by Odijk on the one hand, and by Witten and Pincus on the other hand. While we find that the Witten-Pincus description should be correct for salt free solutions, it also appears that as salt is added, the crossover to Odijk's description is much more rapid than the one described by (3). In fact, we expect this crossover to be very sharp, taking place as soon as the screening length $\kappa^{-1}$ becomes smaller than the interchain distance $D \simeq(c A)^{-1 / 2}$

\section{A "simple" problem: charged chain in two dimensions interacting with point obstacles.}

2.1 PRINCIPLE OF AN EXACT CALCULATION. - We consider a two-dimensional charged wormlike chain, with a bare persistence length $\ell_{0}$. The charged monomers are separated by a distance $A$ along the contour length and interact via a screened Coulomb potential $v_{\mathrm{DH}}(r)=$ $k_{\mathrm{B}} T \ell_{\mathrm{B}} \exp (-\kappa r) / r$. This chain is immersed in a liquid of point particles characterized by its surface density $\Gamma$. Each point particle interacts with the charged monomers via a potential $v_{\text {op }}(r)$, and two point particles interact with each other via a potential $v_{\text {oo }}(r)$. The question to be addressed is that of the effective persistence length of the chain in the presence of the point particles [6]. At the level of Odijk's calculation for the isolated chain, this quantity can be obtained in the following manner. For a given chain configuration which deviates only slightly from a rodlike configuration, the total free energy of the system is computed to second order in the chain curvature $\rho(s)$. Here $s$ is the curvilinear abscissa along the chain, and $\rho(s)$ is assumed to be a small and slowly varying quantity. The coefficient of the second order term is then identified as the effective persistence length of the chain. The free energy is the sum of two terms. The first one is simply the interaction energy between the monomers. Its contribution to the persistence length is the sum of the bare persistence length, $\ell_{0}$, and of the electrostatic persistence length of an isolated chain, $\ell_{\mathrm{OSF}}=\ell_{\mathrm{B}} / 4 \kappa^{2} A^{2}$. The second term is the free energy of the point particles in the field created by the chain. By computing this second term and expanding it to second order in $\rho$, one obtains the desired contribution from the point particles to the effective stiffness of the chain.

The difficult step in this calculation obviously lies in the computation of the free energy $F_{0}$ of the point particles for a given configuration of the chain. One approximate calculation involves an assumption of linear response for the point particles; this results in equation (2). More accurate results can only be obtained for two specific systems, described below.

2.2 EXACT CALCULATION FOR AN IDEAL GAS OF OBSTACLES. - In the case where the point particles form an ideal gas with a given chemical potential, i.e. $v_{\mathrm{oo}}=0$, their free energy can be computed explicitly in terms of the potential $V(\mathbf{r})=\int_{0}^{L} \mathrm{~d} s v_{\mathbf{m o}}(\mathbf{r}(s)-\mathbf{r})$ created by the charged chain in the configuration $\{\mathbf{r}(s)\}$ :

$$
F_{0}=k_{\mathrm{B}} T \Gamma \int \mathrm{d} \mathbf{r}\left(1-\exp \left(-V(\mathbf{r}) / k_{\mathrm{B}} T\right)\right. \text {. }
$$

Of particular interest is the case where the interaction $v_{\text {mo }}$ is of the form $v_{\text {mo }}(r)=2 k_{\mathrm{B}} T\left(\ell_{\mathrm{B}} / A\right)$ $K_{0}(\kappa r)$. Physically, this means that each point particle represents the intersection with the plane in which the chain moves of a charged rod of linear charge density $1 / A$ that lies perpendicular to the plane. A dimensionless coupling constant can then be defined as

$$
b=\frac{2 \pi \ell_{\mathrm{B}}}{\kappa A^{2}}
$$


this is the interaction energy measured in units of $k_{\mathrm{B}} T$ between the chain in a rodlike configuration and an obstacle located very close to the chain. We were able to compute, the free energy of the ideal gas in the field created by a circular chain of radius $R$ up to second order in the curvature $\rho=1 / R$. The free energy can be written in the form:

$$
F=F_{0}+\frac{1}{2} k_{\mathrm{B}} T \frac{\ell_{\mathrm{po}}}{R^{2}}(2 \pi R)
$$

The contribution $\ell_{\text {po }}$ of the obstacles to the persistence length, is given by:

$$
\ell_{\mathrm{po}}=-\Gamma \kappa^{-3} f(b)
$$

where the function $f(b)$ is calculated in Appendix $A$ and reads

$$
f(b)=\frac{b}{2} \int_{0}^{+\infty} \mathrm{d} z \exp (-b \exp (-z))\left[-b z^{2} \exp (-2 z)+\exp (-z)\left(-z^{2}+z+1\right)\right]
$$

In the limit where $b$ is small (strong screening or weak coupling) the linear response theory is valid and $f(b)=3 b^{2} / 8$ in agreement with (2). For large $b$ (weak screening or strong coupling), one gets

$$
\ell_{\mathrm{po}} \simeq-\Gamma \kappa^{-3}(\ln b)^{2}
$$

The interaction with the obstacles thus always reduces the persistence length of the chain. As could be expected, in the strong coupling regime, the decrease of the persistence length depends only very weakly on the Bjerrum length (logarithmically). It has been shown in reference [1] that the Odijk or Skolnick and Fixman theory is valid only if the polymer chain is locally rigid enough. We have assumed here that this condition is satisfied even when the interchain interactions are taken into account.

2.3 EXACT CALCULATION FOR HARD DISK INTERACTIONS. - When the interaction between the point particles and the monomers is a pure hard core repulsion, the contribution of the obstacles to the persistence length vanishes identically. This can be seen from the fact that the free energy of the point particles only depends on the volume excluded by the polymer chain. This excluded volume (or rather surface) is exactly the same for a bent chain and a straight line, provided that the radius of curvature is much larger than the diameter of the hard core interaction. The difference between the hard core interaction and the screened Coulomb interaction is striking, and illustrates the sensitivity of local properties such as the persistence length to the detailed nature of interparticle interactions.

2.4 APPROXIMATE CALCULATION: SUCCESSIVE ANGULAR DEFLECTIONS. - The results of sections 2.2 and 2.3 have the advantage of being essentially exact. They do not, however, give much insight into the physical processes that lead to an increased flexibility of the chain. Worse, they are not easily transferable to the physically relevant three-dimensional situation. In this section, we describe an approximate calculation of the effect of the obstacles, which does not suffer from the same problems. The calculation goes as follows. We consider a charged rodlike chain that passes within a distance $r$ of a fixed obstacle. The minimal energy configuration for this chain is not a straight line, because of the interaction with the obstacle. Instead, the chain is deffected in the vicinity of the obstacle. Let $\theta(r)$ be the angular deflection of the chain (see Fig. 1). This deflection depends on the interaction between the particle and the chain, and on the stiffness (both intrinsic and electrostatic) of the chain. We moreover assume that the 
chain undergoes uncorrelated collisions, and that the probability of finding an obstacle within a distance $r$ from the chain is given by the Boltzmann factor $\exp \left(-V(r) / k_{\mathrm{B}} T\right)$. Under these assumptions, the mean squared angular deflection caused by the obstacles for a contour length $s$ along the chain is

$$
<\theta^{2}(s)>=s \Gamma \int_{0}^{\infty} \mathrm{d} r \exp \left(-V(r) / k_{\mathrm{B}} T\right) \theta^{2}(r)
$$

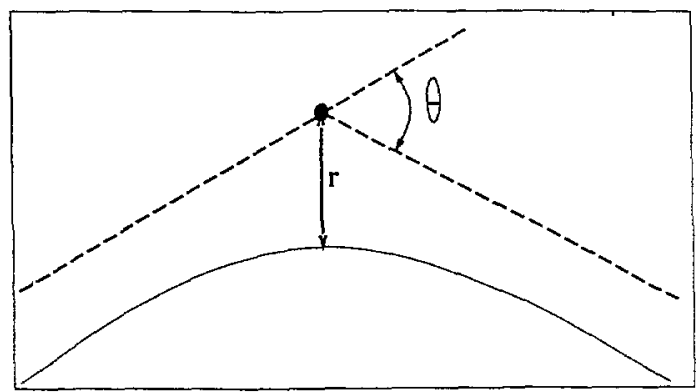

Fig. 1. - Schematic representation of the deflection of a rigid chain by an obstacle.

This deflection adds up to the fluctuations of the orientation that results from the intrinsic and electrostatic rigidities, $s /\left(\ell_{0}+\ell_{\mathrm{OSF}}\right)$. We therefore identify the total persistence length as

$$
\frac{1}{\ell_{\text {tot }}}=\frac{1}{\left(\ell_{0}+\ell_{\text {OSF }}\right)}+\Gamma \int_{0}^{\infty} \mathrm{d} r \exp \left(-V(r) / k_{\mathrm{B}} T\right) \theta^{2}(r)
$$

The angular deflection $\theta(r)$ can be computed if it is small in the case of screened Coulombic interactions considered in section 2.3, $v_{\mathrm{mo}}(r)=2 k_{\mathrm{B}} T \ell_{\mathrm{B}} / A K_{0}(\kappa r)$. We find (see Appendix B):

$$
\theta(r)=\left(\frac{\ell_{\text {OSF }}}{\ell_{0}+\ell_{\text {OSF }}}\right) 8 \pi \kappa r \exp (-\kappa r)
$$

When the electrostatic rigidity is dominant $\left(\ell_{\mathrm{OSF}} \gg \ell_{0}\right)$, this reduces to $\theta(r) \simeq 8 \pi \kappa r \exp (-\kappa r)$ The interaction $V(r)$ between the particle and the (straight) chain, on the other hand, is given by

$$
\begin{aligned}
V(r) & =2 k_{\mathrm{B}} T \frac{\ell_{\mathrm{B}}}{A^{2}} \int_{-\infty}^{+\infty} \mathrm{d} x K_{0}\left(\kappa\left(x^{2}+r^{2}\right)^{1 / 2}\right) \\
& =k_{\mathrm{B}} T b \exp (-\kappa r)
\end{aligned}
$$

Using (11) and (12), the integral over $r$ in equation (10) can be computed as:

$$
\int_{0}^{\infty} \mathrm{d} r \exp \left(-V(r) / k_{\mathrm{B}} T\right) \theta(r)^{2} \simeq 64 \pi^{2} \kappa^{-1} \int_{0}^{\infty} \mathrm{d} u \exp (-b \exp (-u)) u^{2} \exp (-2 u)
$$

and the total persistence length is approximately

$$
\ell_{\mathrm{tot}} \simeq \ell_{\mathrm{OSF}}\left(1+\alpha \frac{\Gamma A^{2}}{\kappa \ell_{\mathrm{B}}} \ln ^{2} b\right)^{-1}
$$


In this last result, we have introduced a numerical coefficient $\alpha$ that accounts for the approximations made in deriving equation (9). Provided that the correction to Odijk's result is not too large, we see that this result agrees with the exact result derived in section 2.2 , equation (8). That the two results agree only to first order in $\Gamma$ is not surprising, since the approximate calculation relies on the assumption that there is no overlap between two successive deflections, an assumption that can be correct only for small obstacle concentrations.

Finally, we note that equation (10) also gives the correct answer in the case of hard core interactions considered in section 2.4. In that case, the deflection is $\theta(r)=0$ for $r>\sigma$, while $\exp \left(-V(r) / k_{\mathrm{B}} T\right)=0$ for $r<\sigma$, so that the integral over $r$ in (10) vanishes.

2.5 NUMERICAL SIMUlations. - It is rather difficult to test these ideas by computer simulations. The difficulty stems from the fact that the calculations are valid, in principle, for infinitely long chains only. End effects are small only if the chain is much longer than the length scale over which it perturbs the liquid of obstacles. For the interaction considered in section 2.2 , and for an ideal gas of obstacles, the perturbation extends over a few screening length $\kappa^{-1}$ (typically, according to $(12),(\ln b) \kappa^{-1}$ ). Therefore, if e.g. $\kappa^{-1}=30 A$ and $\ln (b)=2.5$, a chain of length at least $1000 \mathrm{~A}$ should be used. This chain should be enclosed in a much larger simulation cell containing a correspondingly large number of obstacles (in order to avoid interactions between periodic replicae). The number of particles involved would rapidly become prohibitively large.

One result that can be tested through numerical simulations is the one established in section 2.4 , i.e. that hard obstacles do not affect the persistence length. Figures $2 \mathrm{a}$ and $2 \mathrm{~b}$ display snapshots of a short ( 300 monomers) charged chain immersed in hard disk liquids of various densities. The hard core diameter $\sigma$ is equal to the screening length, $\kappa^{-1}=30 \mathrm{~A}$. A statistical analysis shows that the presence of the hard disks does not modify the angular correlations along the chain, which remain identical to what was described in [1]. Very different is the situation illustrated in figure 3 , where the interaction between the monomers and the point particles is now the "soft" potential $2 k_{\mathrm{B}} T\left(\ell_{\mathrm{B}} / A\right) K_{0}(\kappa r)$, the interaction between the point particles still being a hard-disk interaction. Although in that case end effects preclude a detailed statistical analysis, the configuration of the chain is clearly much more strongly changed by these "soft" obstacles than by the "hard" ones.

\section{Semidilute solutions.}

3.1 ANGULAR DEFLECTION BY A RIGID ROD. - We now consider a three-dimensional solution of charged wormlike chains, similar to that studied in section 2. The concentration of charged monomers is denoted by $c$. The overlap concentration $c^{*}$ is defined as usual as $c^{*}=(L / A) / R^{3}$, where $R$ is the size of the chain in the dilute system and $L$ the contour length. In the semidilute regime $\left(c>c^{*}\right)$, the distance between chains $D$ is defined through $c=(D / A) / D^{3}$, where we imply that the chains are straight rods at scales smaller than $D$. We distinguish between salt-free solutions where $\kappa^{-1}=\left(4 \pi \ell_{\mathrm{B}} c\right)^{-1 / 2} \sim D$, and "strongly screened" solutions, where a concentration $c_{s}$ of monovalent salt is added so that $\kappa^{-1}=\left(4 \pi \ell_{\mathrm{B}}\left(c+2 c_{\mathrm{B}}\right)\right)^{-1 / 2} \ll D$. For salt-free solutions, $c^{*}=1 /\left(A L^{2}\right)$. For screened solutions, we first limit ourselves, in order to simplify the discussion, to relatively short chains, i.e. chains that are rodlike in the dilute regime, so that $c^{*}=1 /\left(A L^{2}\right)$. Longer chains are briefly considered in section 3.4.

Clearly, this three-dimensional problem is much more complex than the two-dimensional one studied in section 2 . It seems reasonable, however, to assume that the influence of the 


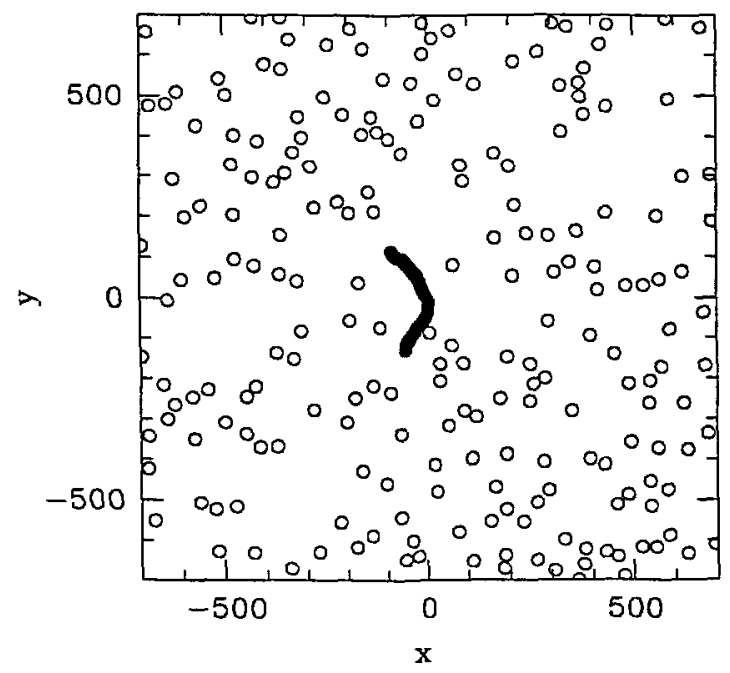

a)

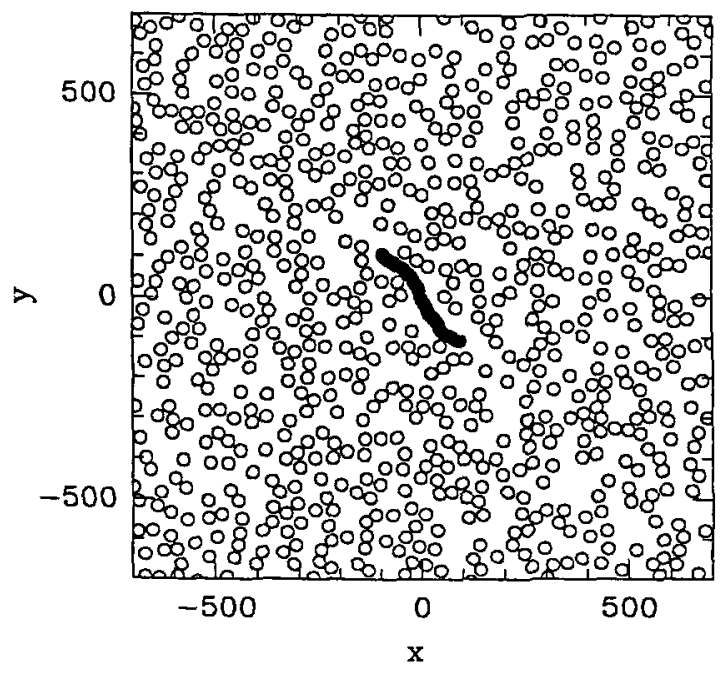

b)

Fig. 2. - a) Snapshot of a typical configuration for a charged polymer chain interacting with obstacles. The chain is a charged wormlike chain characterized by the parameters $A=\ell_{\mathrm{B}}, L=300 A, \ell_{0}=100 A$, $1 / \kappa=30 A$. The obstacles are hard disks of diameter $\sigma=30 A$, the surface fraction occupied by the disks is $\eta=\pi \sigma^{2} \Gamma / 4=0.07$. The monomer-obstacle interaction is of the hard core type, with a diameter $\sigma$. b) Same as figure 2a, except that the surface fraction of the disks is now $\eta=0.28$.

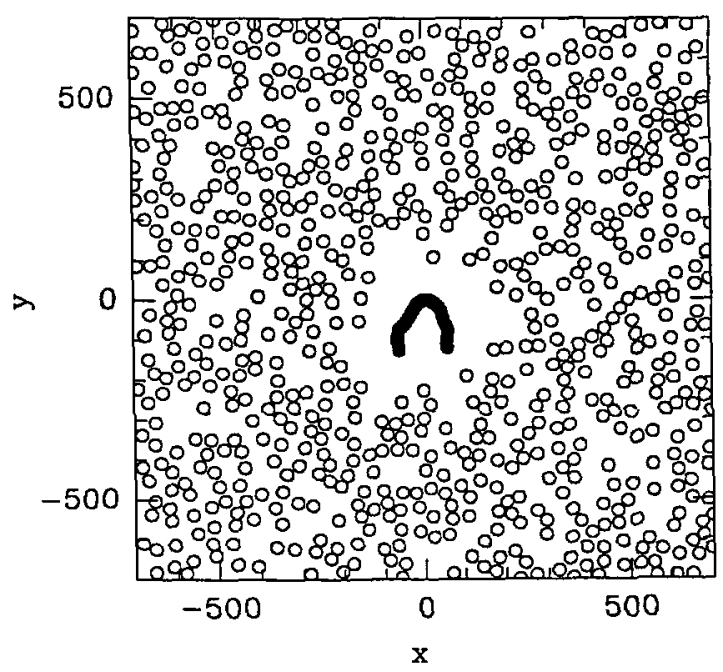

Fig. 3. - Same as figure $2 b$, except the interaction between the monomers and the hard disks is now a soft interaction, $k_{\mathrm{B}} T\left(\ell_{\mathrm{B}} / A\right) K_{0}(\kappa r)$.

other chains on the statistics of one given chain can be described through the same kind of "deflections" that has proved useful in describing the interaction with the obstacles. Two chains interact if they are, locally, within a distance smaller than $\kappa^{-1}$ from one another. The most 
likely configuration for the interaction of two chains is thus the one in which the two chains are, locally, perpendicular straight rods separated by a minimal distance $r$. Other configurations, such as two locally parallel chains, would cost much more in energy, and are therefore very unlikely. More precisely, for two rods separated by a minimal distance $r<\kappa^{-1}$, the energy penalty of a deviation $\delta \theta$ from the perpendicular configuration is of order $k_{\mathrm{B}} T \ell_{\mathrm{B}} \kappa^{-1} A^{-2}(\delta \theta)^{2}$. In the case of interest $\ell_{\mathrm{B}} \kappa^{-1} \gg A^{2}$ and this energy is much larger than $k_{\mathrm{B}} T$ except for very small deviations $(\delta \theta)^{2}<\kappa A^{2} / \ell_{\mathrm{B}}$. We can therefore safely assume that the dominant configurations are the ones where the chains are perpendicular.

The problem of the angular deflection of a charged chain by a perpendicular charged rod is, as mentioned in section 2.2, exactly identical to the problem of the deflection by an obstacle studied in section 2.4, when the interaction $v_{\text {mo }}$ is given by $2 \ell_{\mathrm{B}} / A k_{\mathrm{B}} T K_{0}(\kappa r)$. From these arguments, we conclude that we can use equation (11) to describe the deflection of a given three-dimensional chain by other chains.

The problem is now reduced to the statistics of the angular deflections encountered by a given chain, which determine its persistence length. These statistics are very different for salt-free and for strongly screened solutions; these two cases are considered separately in the following sections.

3.2 THE STRONGLY SCREENED CASE: DEFLECTION BY AN IDEAL GAS OF RIGID RODS. - In the strongly screened case, the semidilute solution can be pictured as an ensemble of wormlike chains with a diameter $\kappa^{-1}$ much smaller than the interchain distance $D$. Each chain has a total persistence length, $\ell_{\text {tot }}$, that results from the balance between the electrostatic interactions inside the chain and the deflections caused by other chains. We assume that the deflections for a tagged chain are the same as those that would be caused by an ensemble of rods of length $L^{\prime} \simeq \ell_{\text {tot }}$, at the same charge concentration as the actual solution. This amounts to neglect the fact that successive sections of size $L^{\prime}$ are tied together in the same chain. In a semidilute solution, where the correlation length is smaller than the chain size, this is a reasonable hypothesis: sections of the same chain that are sufficiently far apart behave as independent entities. A confirmation that this picture is meaningful is given by the fact that within the mean field approximation that we use, the final results do not depend on the precise value of $L^{\prime}$.

The solution of rods equivalent to our original solution has a concentration $c^{\prime}=c A / L^{\prime}$. The tagged chain interacts with this solution of rods. In the spirit of Onsager's calculation for the isotropic-nematic transition, we assume the absence of positional or orientational correlations between the rods (the question of the existence of a nematic order will be addressed below). This means that the solution of rods responds to the perturbation introduced by the tagged chain as an ideal gas. In the absence of interactions, the number of rods lying within a distance $r$ of the tagged chain would be of the order $r L^{\prime} c^{\prime}$ per unit of contour length. This number must be weighed by a Boltzmann factor corresponding to the interaction between the chain and the rod. Following the discussion of section 3.1, we approximate both the Boltzmann factor and the angular deflection by the values appropriate for a chain and a rod in the perpendicular configuration. We certainly overestimate this way the number of rods that deflect the tagged chain, and we probably underestimate the magnitude of the deflections. These approximations are accounted for by a numerical coefficient $\alpha$ in the final formula for the mean squared deflection. After a contour length $s$ along the chain, we find a total change in the orientation

$$
<\theta^{2}(s)>=\alpha s L^{\prime} c^{\prime} \int_{0}^{\infty} \mathrm{d} r \exp \left(-V(r) / k_{\mathrm{B}} T\right) \theta^{2}(r)
$$


The total persistence length is obtained from (15) as:

$$
\ell_{\mathrm{tot}}^{-1}=\ell_{\mathrm{OSF}}^{-1}\left[1+\ln ^{2}(b)\left(\frac{\alpha c A^{3}}{\kappa \ell_{\mathrm{B}}}\right)\right] .
$$

For the screened solutions that we are considering, the second term inside the square brackets is small, since both $\kappa^{-1}$ and $A$ are much smaller than the interchain distance $D=(c A)^{-1 / 2}$ The conclusion is therefore that the persistence length of a chain is only weakly affected by the interchain interactions. Odijk's picture of semidilute solutions should be essentially correct in this case.

An interesting by product of our description in terms of uncorrelated rods is the osmotic pressure of the solution. The osmotic pressure of the solution of rods is given, according to Onsager's theory, by $\Pi \sim k_{\mathrm{B}} T c^{2} L^{\prime 2} \kappa^{-1}$ The regime considered in this section should thus correspond to an osmotic pressure of the polyelectrolyte solution varying as $k_{\mathrm{B}} T c^{2} A^{2} \kappa^{-1}$

3.3 SALT-FREE SOLUTIONS: DEFLECTION BY STRONGLY CORRELATED CHAINS. - The case of salt-free solutions is radically different. Here the interaction range $\kappa^{-1}$ is of the same order of magnitude as the interchain distance $D$, so that a chain is constantly in strong interaction with its neighbours. Strong positional correlations exist between neighbouring chains, which have a preferred distance equal to $D$. The existence of these correlations and of a prefered distance $D$ manifests itself through the existence of a peak at $q_{\max }=2 \pi / D \sim c^{1 / 2}$ in the structure factor of the solution. Very roughly, the solution could be modeled as a set of chains filling all sites on a lattice with lattice spacing $D$. This "lattice" picture strongly suggests that the persistence length of a given chain cannot exceed the lattice spacing $D$ as long as its bare persistence length $\ell_{0}$ is smaller than the lattice spacing $D$. This stems from the fact that the electrostatic rigidity described by Odjik has its origin in purely energetical considerations. The energy of a configuration of the chains on the lattice, however, does not depend on whether the chains are curved at scales larger than $D$ or not. Therefore a rigidity with an energetical origin cannot come into play at scales larger than $D$. Note that the argument here is very similar to Flory's argument concerning the ideality of chains in melts. The electrostatic rigidity, like the excluded volume, is "screened" by the presence of the other chains on a lattice where every site is filled.

A second argument supporting the same conclusion can be given on the basis of equation (11). When applied to two perpendicular chains separated by a distance $\kappa^{-1}$, this equation predicts a deflection of order unity. This means that after a contour length $D$, a chain undergoes a deflection of order unity, since it necessarily has interacted with a neighbouring chain lying at a distance $D \sim \kappa^{-1}$. Therefore the persistence length is smaller than $D$. At scales smaller than $D$, the chain remains rigid due to its own electrostatic interactions, so the persistence length is of order $D \sim \kappa^{-1}$

Finally, we note that the osmotic pressure in the regime considered here should be dominated by the counterions, so that $\Pi \sim c k_{\mathrm{B}} T$.

3.4 CRossover PROBlEMS, DISCUSSION. - The two extreme regimes discussed in sections 3.2 and 3.3 , namely $c_{\mathrm{s}}=0$ and $c_{\mathrm{s}} \gg c$, are separated by a crossover region, where $c_{\mathrm{s}} \sim c$ and the screening length $\kappa^{-1}$ is only slightly smaller than the interchain distance. Experimentally, this crossover could be studied by progressively adding salt to a solution of fixed polymer concentration. The arguments presented in sections 3.2 and 3.3 imply that the persistence length increases very sharply from $\kappa^{-1}$ to $\ell_{\mathrm{OSF}}$ when the added salt concentration $c$ exceeds two or three times the counterions concentration $c$. This can be seen from equation (11). The 
angular deflection caused by a chain located at a distance $D$ from a tagged chain decreases exponentially when the product $\kappa D$ increases. For $c_{\mathrm{s}}=3 c, \kappa D \simeq 10$ and $\theta(D) \simeq 0.02$. This means that the chains located at the average interchain distance become very inefficient at deflecting the tagged chain. The tagged chain is thus deflected only by other chains that approach it within the range $\kappa^{-1}$, and the statistics of these -rare - events is correctly described by the theory presented in section 3.2. Thus for $c_{\mathrm{s}}>3 c$ we can expect $\ell_{\text {tot }} \simeq \ell_{\text {OSF }}$. For $c_{\mathrm{s}}<c$ on the other hand the screening is dominated by the counterions, and $\ell_{\text {tot }}=D \sim \kappa^{-1}$. We thus predict, like Witten and Pincus, an increase in the persistence length as salt is added to the solution. Their original prediction, however, was of a smooth increase, with a persistence length given by (3), and is thus very different from ours (see, however, [7]). Finally, we also note that the osmotic pressure has a sharp crossover when $D \simeq \kappa^{-1}$ If the interchain distance $D$ is smaller than the screening length, the counterions clouds of different chains overlap and the counterions are roughly uniformly distributed, the osmotic pressure is that of a perfect gas of counterions. When $D$ becomes larger than $\kappa^{-1}$, the counterions clouds no longer overlap and the osmotic pressure decays steeply as $\exp (-\kappa D)$. When the screening length is much smaller than the interchain distance, the interaction between chains is equivalent to a hard sphere interaction and the Onsager theory for hard rods can be used.

Another relevant crossover is the crossover between dilute and semidilute solutions. For salt-free solutions, the situation is simple: for $c<c^{*}$, the polymers are practically rodlike objects. Both $\ell_{\mathrm{OSF}}$ and $\kappa^{-1}$ are larger than the chain length. When $c$ increases beyond $c^{*}$, the persistence length starts to decrease as $c^{-1 / 2}$, and the polymers become progressively more coiled. For short chains $\left(L<\ell_{\text {OSF }}\right.$ ) in a screened system, on the other hand, the persistence length does not change at $c^{*}$. Finally, for long chains in a screened system, the semidilute regime must be divided into two regions: at low concentrations, the correlation length is larger than $\ell_{\text {OSF }}$, so that we have a semidilute solution of flexible polymers with a persistence length $\ell_{\text {OSF. }}$. At higher concentrations, the correlation length becomes smaller than $\ell_{O S F}$, and the description of section 3.2 applies.

\section{Concluding remarks.}

In reference [1] two kinds of polyelectrolytes were identified. Flexible polyelectrolytes have a small intrinsic rigidity or a small charge density. In a salt solution the persistence length of isolated flexible polyelectrolytes is of the order of the electrostatic screening length as first proposed by Katchalsky [8]. Rigid polyelectrolytes have a large intrinsic rigidity and a large charge density and accurately described by the theory of Odijk [2] or Skolnick and Fixman [3], their persistence length is larger than the screening length.

Flexible polyelectrolytes thus only have one characteristic length scale and it seems reasonable to study them by constructing scaling laws. This was done some time ago by Pfeuty [9] and de Gennes et al. [8]. In the absence of salt, the correlation length of the solution, the persistence length of the chains and the electrostatic screening length are all of the same order of magnitude and scale with the monomer concentration as $c^{-1 / 2}$

For rigid polyelectrolytes, there are several relevant length scales, the screening length, the distance between chains and the persistence length. The arguments presented in this paper, in agreement with those of Witten and Pincus, show that in a semidilute solution in the absence of added salt the three length scales also become equal and scale as $c^{-1 / 2}$. This is the same result as for flexible chains; the intrinsic rigidity of the chains therefore does not seem to be important for the properties of salt-free semidilute solutions.

As salt is added, we predict a sharp increase of the persistence length when the density of 
the salt becomes larger than the density of counterions, followed by a slow decrease described by Odijk's theory. In the strongly screened regime the persistence length is larger than both the screening length and the distance between chains and does not depend significantly on interchain interactions.

All the results obtained in this work have been discussed at the level of scaling laws where the numerical prefactors are supposed to be of order unity. In some cases however large numerical prefactors or logarithmic corrections seem to appear such as in equations $(13,16)$. We do not believe that they will have any effect on the scaling regime but their precise values may control the width of the crossover regimes which have only been discussed qualitatively here.

Another important limitation is that we have assumed throughout that the polyelectrolyte solution is an isotropic liquid. Two types of ordering may occur but do not seem to be observed experimentally. Just below the overlap concentration, in the absence of salt, one may expect the formation of a crystalline phase where the positions of the centers of mass of the macromolecules are on the sites of a periodic lattice. The existence of this crystalline phase would not affect our results qualitatively as explained in section 3.3. For strongly screened solutions, the chains become very anisotropic and may undergo an isotropic to nematic transition. The critical concentration for this transition is of the order of the Onsager concentration for rods with a length equal to the persistence length $\ell_{\mathrm{OSF}}$ (when the electrostatic persistence length is larger than the intrinsic persistence length) and a diameter of the order of $d=\kappa^{-1} \ln b: c_{0}=$ $\kappa^{3} A / \ell_{\mathrm{B}} \ln b$. At constant polymer concentration, one would thus expect nematic ordering as soon as the salt concentration $c_{\mathrm{s}}$ is larger than the counterion concentration $c$ and smaller than the value where the polymer concentration becomes equal to the Onsager value. Experimentally no nematic ordering is observed (except when the intrinsic persistence length is very large). This may be due to the fact that a section of chain of size $\ell_{O S F}$ is not very anisotropic: its asymmetry ratio is $\alpha=\ell_{\mathrm{OSF}} / d=b /(8 \pi \ln b)$. Computer simulations [10] show that a nematic phase is observed if the asymmetry ratio is larger than 4-5; this would thus require a prohibitively large value of the coupling constant $b$.

\section{Acknowledgements.}

Useful correspondence and discussions with Phil Pincus and Tom Witten are gratefully acknowledged.

\section{Appendix A.}

\section{Ideal gas of obstacles in two dimensions.}

In this appendix we explicitly calculate the free energy of an ideal gas of obstacles interacting with a two-dimensional curved chain. The test chain is bent on a circle of radius $R$ and interacts with the obstacles via a screened Coulomb potential. The obstacles can be viewed as infinite rodlike chains perpendicular to the plane of the test chain. We consider one obstacle at a distance $c$ from the curved chain. We measure the position of a point on the obstacle by the curvilinear abscissa $\ell^{\prime}$ where the origin is taken in the plane and on the tagged chain by the curvilinear abscissa $\ell$ the origin being taken at the point of minimal distance between the tagged chain and the perpendicular obstacle. The electrostatic interaction energy between the two polymers is

$$
V(c) / k_{\mathrm{B}} T=\ell_{\mathrm{B}} A^{-2} \int_{-\infty}^{+\infty} \mathrm{d} \ell \mathrm{d} \ell^{\prime} \frac{\exp (-\kappa d)}{d}
$$


In the limit where the radius $R$ is large, the distance $d$ between points at positions $\ell$ and $\ell^{\prime}$ can be expanded in powers of the curvature $1 / R$ to second order.

$$
d^{2}=c^{2}+\ell^{2}+\ell^{\prime 2}+\frac{c \ell^{2}}{R}-\frac{\ell^{4}}{12 R^{2}}
$$

It is then convenient to introduce the distance $\rho$ and the angle $\theta$ such that $\ell=\rho \cos \theta$ and $\ell^{\prime}=\rho \sin \theta$. The electrostatic interaction energy can be written as

$$
V(c) / k_{\mathrm{B}} T=\ell_{\mathrm{B}} A^{-2} \int \mathrm{d} \theta \rho d \rho \frac{\exp (-\kappa d)}{d}
$$

This integral is calculated by changing variable from $\rho$ to $d$

$$
V(c) / k_{\mathrm{B}} T=2 \pi \ell_{\mathrm{B}} A^{-2} \int_{|c|}^{+\infty} \mathrm{d} d \exp (-\kappa d)\left[1-\frac{c<\cos \theta>}{R}+\frac{\left(d^{2}+5 c^{2}\right)<\cos ^{4} \theta>}{6 R^{2}}\right]
$$

where the brackets denote an average over the angle $\theta$. The interaction potential can be recast as $V(c) / k_{\mathrm{B}} T=V_{0}+\left(V_{1} / R\right)+\left(V_{2} / R^{2}\right)$ with $V_{0}=b \exp (-\kappa|c|), V_{1}=-c b / 2 \exp (-\kappa|c|)$ and and $V_{2}=\exp (-\kappa|c|)\left[3 c^{2} / 8+|c| /(8 \kappa)+1 /\left(8 \kappa^{2}\right)\right]$.

The free energy of the gas of obstacles (4) can then be expanded in powers of the curvature; the second order term which gives the persistence length reads

$$
F_{2} / k_{\mathrm{B}} T=\Gamma / R\left[\int_{-\infty}^{+\infty} 2 \pi c \mathrm{~d} c V_{1} \exp \left(-V_{0}\right)+\int_{-\infty}^{+\infty} 2 \pi \mathrm{d} c\left(-V_{1}^{2} / 2+V_{2}\right) \exp \left(-V_{0}\right)\right]
$$

The calculation of this integral gives the result of equation (7) in the text for the persistence length.

\section{Appendix B.}

\section{Calculation of the angular deflection.}

In this appendix, the computation of the angular deflection of a chain by an obstacle in two dimensions (Eq. (11)) is briefly described. The geometry is illustrated in figure 1. Taking the tangent to the chain at the point of closest approach as the $x$, we can parametrize the chain contour as:

$$
\begin{aligned}
& x(s)=\int_{0}^{s} \mathrm{~d} s^{\prime} \cos \left(\theta\left(s^{\prime}\right)\right) \simeq s-\frac{1}{2} \int_{0}^{s} \mathrm{~d} s^{\prime} \int_{0}^{s^{\prime}} \mathrm{d} s_{1} \int_{0}^{s^{\prime}} \mathrm{d} s_{2} \frac{\mathrm{d} \theta}{\mathrm{d} s_{1}} \frac{\mathrm{d} \theta}{\mathrm{d} s_{2}} \\
& y(s)=\int_{0}^{s} \mathrm{~d} s^{\prime} \sin \left(\theta\left(s^{\prime}\right)\right) \simeq \int_{0}^{s} \mathrm{~d} s^{\prime} \int_{0}^{s^{\prime}} \mathrm{d} s_{1} \frac{\mathrm{d} \theta}{\mathrm{d} s_{1}}
\end{aligned}
$$

where $s$ is the curvilinear abscissa along the chain and $(\cos (\theta(s)), \sin (\theta(s)))$ defines the tangent vector at abscissa $s$.

The principle of the calculation is to write the interaction with the obstacle in the form

$$
E_{\text {int }}=\text { Cte }+\int \mathrm{d} s g(s) \frac{\mathrm{d} \theta}{\mathrm{d} s}
$$


and to balance this energy against the curvature energy

$$
E_{\text {curv }}=\frac{1}{2}\left(\ell_{0}+\ell_{\text {OSF }}\right) \int \mathrm{d} s\left(\frac{\mathrm{d} \theta}{\mathrm{d} s}\right)^{2}
$$

Here we have made the simplifying assumption (see [1]) that the electrostatic stiffness can be approximated by a local curvature term. This balance yields for the deflection

$$
\theta(r)=\int_{-\infty}^{+\infty}-2 \mathrm{~d} s \frac{\mathrm{d} \theta}{\mathrm{d} s}=\frac{1}{\ell_{0}+\ell_{\mathrm{OSF}}} \int_{0}^{+\infty} \mathrm{d} s g(s)
$$

In order to cast the interaction energy into the desired form (Eq. (23)), we expand the interaction between a monomer and the obstacle using:

$$
\begin{aligned}
\left.K_{0}\left(\kappa\left[x(s)^{2}+(y(s)+r)^{2}\right)\right]^{1 / 2}\right) & =K_{0}\left(\kappa\left[s^{2}+r^{2}\right]^{1 / 2}\right) \\
& -\kappa K_{1}\left(\kappa\left[s^{2}+r^{2}\right]^{1 / 2}\right) \frac{r}{\left[s^{2}+r^{2}\right]^{1 / 2}} \int_{0}^{s} \mathrm{~d} s^{\prime}\left(s-s^{\prime}\right) \frac{\mathrm{d} \theta}{\mathrm{d} s^{\prime}} .
\end{aligned}
$$

The interaction energy becomes

$$
\begin{aligned}
E_{\text {int }}= & E_{\text {int }} \text { (rodlike configuration) } \\
& -2 k_{\mathrm{B}} T \ell_{\mathrm{B}} \kappa A^{-2} \int_{-\infty}^{+\infty} \mathrm{d} s \frac{r}{\left[s^{2}+r^{2}\right]^{1 / 2}} K_{1}\left(\kappa\left[s^{2}+r^{2}\right]^{1 / 2}\right) \int_{0}^{s} \mathrm{~d} s^{\prime}\left(s-s^{\prime}\right) \frac{\mathrm{d} \theta}{\mathrm{d} s^{\prime}}
\end{aligned}
$$

which can be written in the form (23) with

$$
g(s)=2 k_{\mathrm{B}} T \ell_{\mathrm{B}} \kappa A^{-2} \int_{s}^{+\infty} \mathrm{d} s^{\prime} \frac{r\left(s-s^{\prime}\right)}{\left[s^{2}+r^{2}\right]^{1 / 2}} K_{1}\left(\kappa\left[s^{2}+r^{2}\right]^{1 / 2}\right) .
$$

The integral appearing in equation (25) can finally be obtained using the following transformations:

$$
\begin{aligned}
\int_{0}^{+\infty} \mathrm{d} s g(s) & =-4 \kappa \ell_{\mathrm{B}} A^{-2} \int_{0}^{+\infty} \mathrm{d} s \int_{0}^{+\infty} \mathrm{d} t \frac{r t}{\left[r^{2}+(s+t)^{2}\right]^{1 / 2}} K_{1}\left(\kappa\left[r^{2}+(s+t)^{2}\right]^{1 / 2}\right) \\
& =-4 \kappa \ell_{\mathrm{B}} A^{-2} \int_{0}^{+\infty} \mathrm{d} u \frac{u^{2}}{\left[r^{2}+u^{2}\right]^{1 / 2}} K_{1}\left(\kappa\left[r^{2}+u^{2}\right]^{1 / 2}\right)
\end{aligned}
$$

To obtain the second line in (29), we have used the transformation $u=s+t, t=\xi u$ and integrated over $\xi$. Using the integral representation of $K_{1}$, we can transform this formula into

$$
\begin{aligned}
\int_{0}^{+\infty} \mathrm{d} s g(s) & =-2 \ell_{\mathrm{B}} A^{-2} r^{-1} \int_{0}^{+\infty} \mathrm{d} t \int_{0}^{+\infty} \mathrm{d} u \frac{u^{2}}{\left[t^{2}+1+u^{2} / r^{2}\right]^{3 / 2}} \cos (\kappa r t) \\
& =-2 \ell_{\mathrm{B}} r^{2} A^{-2} \int_{0}^{+\infty} \rho \mathrm{d} \rho \int_{0}^{2 \pi} \mathrm{d} \phi \frac{\rho^{2}}{\left(1+\rho^{2}\right)^{3 / 2}} \sin ^{2}(\phi) \cos (\kappa r \rho \cos (\phi))
\end{aligned}
$$

Here the transformations $x=u / r=\rho \sin \phi$ and $t=\rho \cos \phi$ have been used. Finally, using formulae (9.120) and (11.444) in [11], one has:

$$
\begin{aligned}
\int_{0}^{+\infty} \mathrm{d} s g(s) & =-2 \ell_{\mathrm{B}} r^{2} A^{-2} \int_{0}^{+\infty} \rho \mathrm{d} \rho \frac{\rho^{2}}{\left(1+\rho^{2}\right)^{3 / 2}} J_{1}(\kappa r \rho) \\
& =(8 \pi)^{1 / 2} \ell_{\mathrm{B}} r \kappa^{-1} A^{-2}(\kappa r)^{1 / 2} K_{1 / 2}(\kappa r)
\end{aligned}
$$

which combined with (25) yields (11). 


\section{References}

[1] Barrat J.-L. and Joanny J.-F., Europhys. Lett. 24 (1993) 333.

[2] Odijk T., J. Polym. Sci 15 (1977) 477.

[3] Skolnick J. and Fixman M., Macromolecules 10 (1977) 944.

[4] Odijk T., Macromolecules 12 (1979) 688.

[5] Witten T.A. and Pincus P., Europhys. Lett. 3 (1987) 315.

[6] This question is different from the usual, well-understood problem of the large scale properties (Flory exponent) of a polymer chain immersed in a solvent. For the large scale properties, it is well-known that the effect of the solvent can be accounted for by introducing an effective excluded volume between monomers, which can be either positive or negative depending on the mismatch between monomer and solvent. This approach is, however, not sufficient for studying properties of the chain at small (or intermediate) scales, such as the local rigidity we are interested in.

[7] Witten T. and Pincus P., J. Phys. II France 4 (1994) 1103. In this note, Witten and Pincus present a revised version of their theory, which also predicts a sharp crossover between the two regimes and from which the intermediate length $\ell_{\mathrm{WP}}$ is absent.

[8] de Gennes P.G., Pincus P., Velasco R.M., Brochard F., J. Phys. France 37 (1976) 1461.

[9] Pfeuty P., J. Phys. Colloq. France 39 (1978) C2-149.

[10] Frenkel D., Lekkerkerker H. and Stroobants A., Nature 332 (1988) 822.

[11] Abramovitz M. and Stegun I.A., Handbook of mathematical functions (Dover, 1965). 